\title{
$\begin{array}{ll}\text { Research Square } & \text { Preprints are preliminary reports that have not undergone peer review. } \\ \text { They should not be considered conclusive, used to inform clinical practice, }\end{array}$ or referenced by the media as validated information. \\ Influence of reinforcing ribs on the deformed state of ellipsoidal shells under distributed load
}

Nataliia Maiborodina ( $\nabla$ natashamai2311@gmail.com )

Separated Subdivision NULESU "Nizhyn Agrotechnical Institute" https://orcid.org/0000-0003-17546790

\section{Viacheslav Gerasymenko}

Separated Subdivision NULESU "Nizhyn Agrotechnical Institute"

\section{Oleksandr Kovalov}

Dmytro Motornyi Tavria State Agrotechnological University

\section{Research Article}

Keywords: Oscillations, Shell, Discretely reinforced, Non-stationary load, Numerical algorithm

Posted Date: November 22nd, 2021

DOI: https://doi.org/10.21203/rs.3.rs-1057386/v1

License: (c) (i) This work is licensed under a Creative Commons Attribution 4.0 International License. Read Full License 


\title{
Influence of reinforcing ribs on the deformed state of ellipsoidal shells under distributed load
}

\author{
Nataliia Maiborodina https://orcid.org/0000-0003-1754-6790 \\ Viacheslav Gerasymenko https://orcid.org/0000-0002-4017-1141 \\ Oleksandr Kovalov https://orcid.org/ 0000-0002-5822-5494
}

\begin{abstract}
This paper presents the problem of nonstationary oscillations of reinforced ellipsoidal shells, taking into account the discrete location of the ribs. Problem bases on a geometrically nonlinear variant of the Tymoshenko theory for shells and rods. A numerical method for solving problems of this class has been developed and substantiated. This article focuses on the location of the reinforcing ribs. Based on the developed numerical method the deformed state of discretely supported ellipsoidal shells for internal, external, and internal-external placement of ribs is investigated. Boundary conditions for rigidly clamped edges of the shell were studied.
\end{abstract}

Keywords Oscillations - Shell - Discretely reinforced Non-stationary load - Numerical algorithm.

\section{Introduction}

Thin-walled structural elements in the form of shells with various shapes are widely used in mechanical engineering, construction, aerospace, shipbuilding. The shell elements, which consist of the shell itself and reinforcing ribs, have become widespread. The method of fastening the ribs ensures their joint work.

When calculating the strength of reinforced shell structures under dynamic loads, it is necessary to determine the deformed state both in the field of changes in the physical and mechanical parameters for the structure, and at a considerable distance from the inhomogeneities. In this case, one of the features of the studied process is the wave nature and nonlinear distribution of physical and mechanical parameters throughout the system. The complexity of the resulting processes necessitates the use of modern numerical methods for solving dynamic problems of shell elements with reinforcing ribs.

\footnotetext{
N. V. Maiborodina $(\bowtie)$

Department Of Natural Mathematical And General Engineering Disciplines, Separated subdivision of National University of Life And Environmental Sciences of Ukraine «Nizhin Agrotechnical Institute», Shevchenko street, 10 , Nizhyn, Chernihiv region, 16600, Ukraine

e-mail: natashamai2311@gmail.com

V. P. Gerasymenko

Department Of Electric Power Engineering, Electrical Engineering And Electromechanics, Separated Subdivision Of National University Of Life And Environmental Sciences Of Ukraine «Nizhin Agrotechnical Institute», Shevchenko street, 10, Nizhyn, Chernihiv region, 16600, Ukraine e-mail: syavagvp@gmail.com

O. V. Kovaloy

Department Of Theoretical And General Electrical Engineering, Dmytro Motorny Tavriya State Agrotechnological University, B. Khmelnytsky Avenue, 18, Melitopol, Zaporizhia region, 72312, Ukraine

e-mail: alekstdaty1979@gmail.com
}

According to reviews and monographs, harmonic oscillations for reinforced shells of simple geometry (cylindrical, conical, and spherical) were mainly considered [1- 3, 11 - 12, 17]. Forced oscillations of reinforced shells under pulse loads were studied in $[4-5,10,14,16]$. There is almost no work to study the dynamic behavior of reinforced shells with more complex shapes. In this direction, we can distinguish the following works [6 - 9], which present the results of forced oscillations for the shells of rotation in particular ellipsoidal reinforced shells.

In this paper, the equations for non-axisymmetric oscillations of a discretely reinforced ellipsoidal shell are presented. When considering the shell and reinforcing ribs, a refined model for shells and rods of the Tymoshenko type is used [5]. The Hamilton-Ostrogradsky variational principle is used to derive the equations of oscillations. Such problems are quite difficult to set and solve. We use a numerical method based on the application of the integrationinterpolation method to construct difference schemes for equations with discontinuous coefficients.

The goal is to study the influence of the location reinforcing ribs on the deformed state of ellipsoidal shells under distributed load.

\section{Statement of the problem}

We consider an ellipsoidal shell of constant thickness $h$. The shell is attributed to the Gaussian curvilinear coordinate system $\alpha_{1}, \alpha_{2}$.

The middle surface of a smooth ellipsoidal shell is given by the relations:

$$
\begin{gathered}
x=R \sin \alpha_{1} \sin \alpha_{2} ; \\
y=R \sin \alpha_{1} \cos \alpha_{2} ; \\
z=k R \cos \alpha_{1},
\end{gathered}
$$

where $k=b / a$ - ellipticity parameter, $a, b-$ the half axis of the ellipse.

To build a mathematical model for the deformation of the reinforced ellipsoidal shell, we will use a geometrically nonlinear version of the Tymoshenko-type theory for shells in the quadratic approximation [5].

The change in displacement along the thickness of the shell is given in the form:

$$
\begin{gathered}
u_{1}^{z}\left(s_{1}, s_{2}, z\right)=u_{1}\left(s_{1}, s_{2}\right)+z \varphi_{1}\left(s_{1}, s_{2}\right) \\
u_{2}^{z}\left(s_{1}, s_{2}, z\right)=u_{2}\left(s_{1}, s_{2}\right)+z \varphi_{2}\left(s_{1}, s_{2}\right) \\
u_{3}^{z}\left(s_{1}, s_{2}, z\right)=u_{3}\left(s_{1}, s_{2}\right)
\end{gathered}
$$$$
\text { where } \quad z \in[-h / 2, h / 2] \quad s_{1}=\alpha_{1} A_{1}, \quad s_{2}=\alpha_{2} A_{2}, A_{1}, A_{2}-
$$

coefficients of the first quadratic form for the middle surface of the given ellipsoidal shell.

Deformation relations for a smooth shell according [13]: 


$$
\begin{array}{ll}
\varepsilon_{11}=\frac{\partial u_{1}}{\partial s_{1}}+k_{1} u_{3}+\frac{1}{2} \theta_{1}^{2}, & \\
\varepsilon_{22}=\frac{\partial u_{2}}{\partial s_{2}}+\frac{1}{A_{2}} \frac{\partial A_{2}}{\partial s_{1}} u_{1}+k_{2} u_{3}+\frac{1}{2} \theta_{1}^{2}, & \varepsilon_{13}=\varphi_{1}+\theta_{1}, \\
\varepsilon_{12}=\omega+\theta_{1} \theta_{2}, & \omega=\omega_{1}+\omega_{2}, \\
\varepsilon_{23}=\varphi_{2}+\theta_{2}, & \omega_{2}=\frac{\partial u_{1}}{\partial s_{2}}-\frac{1}{A_{2}} \frac{\partial A_{2}}{\partial s_{1}} u_{2}, \\
\omega_{1}=\frac{\partial u_{2}}{\partial s_{1}}, & \theta_{2}=\frac{\partial u_{3}}{\partial s_{2}}-k_{2} u_{2}, \\
\theta_{1}=\frac{\partial u_{3}}{\partial s_{1}}-k_{1} u_{1}, & \chi_{22}=\frac{\partial \varphi_{2}}{\partial s_{2}}+\frac{1}{A_{2}} \frac{\partial A_{2}}{\partial s_{1}} \varphi_{1} \\
\chi_{11}=\frac{\partial \varphi_{1}}{\partial s_{1}}, & \tau_{1}=\frac{\partial \varphi_{2}}{\partial s_{1}}, \\
\chi_{12}=\tau_{1}+\tau_{2}+\kappa_{1} \omega_{1}+\kappa_{2} \omega_{2}, & \\
\tau_{2}=\frac{\partial \varphi_{1}}{\partial s_{2}}-\frac{1}{A_{2}} \frac{\partial A_{2}}{\partial s_{1}} \varphi_{2} . &
\end{array}
$$

Expressions for the forces and moments of the smooth ellipsoidal shell have the form:

$$
\begin{array}{ll}
T_{11}=B_{11} \varepsilon_{11}+B_{12} \varepsilon_{22}, & T_{22}=B_{21} \varepsilon_{11}+B_{22} \varepsilon_{22}, \\
T_{12}=S+k_{2} H, & T_{21}=S+k_{1} H \\
T_{13}=B_{13} \varepsilon_{13}, & T_{23}=B_{23} \varepsilon_{23}, \\
S=B_{s} \varepsilon_{12}, & M_{11}=D_{11} \chi_{11}+D_{12} \chi_{22}, \\
M_{22}=D_{21} \chi_{11}+D_{22} \chi_{22}, & M_{12}=M_{21}=H, \\
H=D_{s} \chi_{12}, &
\end{array}
$$

where

$$
\begin{array}{ll}
D_{s}=G_{12} h^{3} / 12, & B_{s}=G_{12} h, \\
B_{11}=\frac{E_{1} h}{1-v_{12} v_{21}}, & B_{12}=\frac{E_{1} v_{21} h}{1-v_{12} v_{21}}, \\
B_{21}=\frac{E_{2} v_{12} h}{1-v_{12} v_{21}}, & B_{22}=\frac{E_{2} h}{1-v_{12} v_{21}}, \\
B_{13}=G_{13} h k^{2}, & B_{23}=G_{23} h k^{2}, \\
D_{11}=\frac{E_{1} h^{3}}{12\left(1-v_{12} v_{21}\right)}, & D_{12}=\frac{E_{1} v_{21} h^{3}}{12\left(1-v_{12} v_{21}\right)}, \\
D_{21}=\frac{E_{2} v_{12} h^{3}}{12\left(1-v_{12} v_{21}\right)}, & D_{22}=\frac{E_{2} h^{3}}{12\left(1-v_{12} v_{21}\right)} .
\end{array}
$$

In formulas (5) the values of $E_{1}, E_{2}, G_{12}, G_{13}, G_{23}, v_{12}$, $v_{21}$ - physical characteristics of the shell material; $k^{2}-$ the shear coefficient for the shell.
We accept the following hypothesis to build a mathematical model of deformation for the $j$-th reinforcing rib, directed along the axis $\alpha_{2}$. The cross-section of the rib is not deformed. We use the geometrically nonlinear theory of Tymoshenko's rods.

The change in displacements along the cross-section of the $j$-th reinforcing rib is given by an approximation:

$$
\begin{gathered}
u_{1 j}^{x z}\left(x, s_{2}, z\right)=u_{1 j}\left(s_{2}\right)+z \varphi_{1 j}\left(s_{2}\right), \\
u_{2 j}^{x z}\left(x, s_{2}, z\right)=u_{2 j}\left(s_{2}\right)+z \varphi_{2 j}\left(s_{2}\right), \\
u_{3 j}^{x z}\left(x, s_{2}, z\right)=u_{3 j}\left(s_{2}\right),
\end{gathered}
$$

where $u_{1 j}, u_{2 j}, u_{3 j}, \varphi_{1 j}, \varphi_{2 j}$ are the components for the generalized vector of displacements for the cross-section gravity center of the $j$-th rib.

We assume that the shell and discrete reinforcing elements are rigidly connected.

The contact conditions between the components of generalized displacement vector for the cross-sections gravity centers of the $j$-th rib, which is directed along the axis $\alpha_{2}$, and the components of generalized displacement vector for the original middle surface of the smooth ellipsoidal shell have the form:

$$
\begin{aligned}
& u_{1 j}\left(s_{2}\right)=u_{1}\left(s_{1 j}, s_{2}\right) \pm h_{c j} \varphi_{1}\left(s_{1 j}, s_{2}\right), \\
& u_{2 j}\left(s_{2}\right)=u_{2}\left(s_{1 j}, s_{2}\right) \pm h_{c j} \varphi_{2}\left(s_{1 j}, s_{2}\right), \\
& u_{3 j}\left(s_{2}\right)=u_{3}\left(s_{1 j}, s_{2}\right), \\
& \varphi_{1 j}\left(s_{2}\right)=\varphi_{1}\left(s_{1 j}, s_{2}\right), \\
& \varphi_{2 j}\left(s_{2}\right)=\varphi_{2}\left(s_{1 j}, s_{2}\right),
\end{aligned}
$$

where $h_{c j}=0,5\left(h+h_{j}\right)$ - distance from the middle surface of the smooth ellipsoidal shell to the line for the centers of gravity for the cross-sections for $j$-th rib; $h_{j}$ - the height of the reinforcing $j$-th rib; $s_{1 j}$ - coordinate for the design line of the cross-sections gravity centers of the $j$-th rib on the coordinate middle surface of the shell.

The sign "+" corresponds to the case of external ribs, and the sign "-" is chosen in the case of internal ribs.

Deformation relations for the $j$-th rib according to [13]:

$$
\begin{aligned}
& \varepsilon_{22 j}=e_{22 j}, \quad e_{22 j}=\frac{\partial u_{2}}{\partial s_{2}} \pm h_{c j} \frac{\partial \varphi_{2}}{\partial s_{2}}+k_{2 j} u_{3}, \\
& \varepsilon_{21 j}=\theta_{2 j}, \quad \varepsilon_{23 j}=\varphi_{2}+\theta_{1 j}, \\
& \theta_{1 j}=\frac{\partial u_{3}}{\partial s_{2}}-k_{2 j}\left(u_{2} \pm h_{c j} \varphi_{2}\right), \quad \theta_{2 j}=\frac{\partial u_{1}}{\partial s_{2}} \pm h_{c j} \frac{\partial \varphi_{1}}{\partial s_{2}}, \\
& \chi_{21 j}=\frac{\partial \varphi_{1}}{\partial s_{2}}, \quad \chi_{22 j}=\frac{\partial \varphi_{2}}{\partial s_{2}} .
\end{aligned}
$$

Expressions for the forces and moments for the $j$-th rib have the form:

$$
\begin{array}{ll}
T_{21 j}=G_{j} F_{j} \varepsilon_{21 j}, & T_{22 j}=E_{j} F_{j} \varepsilon_{22 j}, \\
T_{23 j}=G_{j} F_{j} k_{j}^{2} \varepsilon_{23 j}, & M_{21 j}=G_{j} I_{c r j} \chi_{21 j},
\end{array}
$$


$M_{22 j}=E_{j} I_{2 j} \chi_{22 j}$.

In formulas (9) the value of $k_{j}^{2}$ - the shear coefficient for the reinforcing rib; $F_{j}, I_{2 j}, I_{c r j}-$ geometric and physical characteristics of transverse elements.

\section{Equation of oscillations}

The Hamilton-Ostrogradsky variational principle is used to derive oscillation equations for a discretely supported structure. We obtain two groups of equations after performing the operations of variation and integration, taking into account the contact conditions (7).

The equations of the shell in a smooth region:

$\frac{1}{A_{2}}\left[\frac{\partial}{\partial s_{1}}\left(A_{2} T_{11}\right)-\frac{\partial A_{2}}{\partial s_{1}} T_{22}\right]+k_{1} \bar{T}_{13}+\frac{1}{A_{1}} \frac{\partial}{\partial s_{2}}\left(A_{1} T_{21}\right)=\rho h \frac{\partial^{2} u_{1}}{\partial t^{2}} ;$

$\frac{1}{A_{2}}\left[\frac{\partial}{\partial s_{1}}\left(A_{2} T_{12}\right)-\frac{\partial A_{2}}{\partial s_{1}} T_{21}\right]+k_{2} \bar{T}_{23}+\frac{1}{A_{1}} \frac{\partial}{\partial s_{2}}\left(A_{1} T_{22}\right)=\rho h \frac{\partial^{2} u_{2}}{\partial t^{2}}$;

$\frac{1}{A_{2}} \frac{\partial}{\partial s_{1}}\left(A_{2} \bar{T}_{13}\right)-k_{1} T_{11}-k_{2} T_{22}+P_{3}+\frac{1}{A_{1}} \frac{\partial}{\partial s_{2}}\left(A_{1} \bar{T}_{23}\right)=\rho h \frac{\partial^{2} u_{3}}{\partial t^{2}} ;$

$\frac{1}{A_{2}}\left[\frac{\partial}{\partial s_{1}}\left(A_{2} M_{11}\right)-\frac{\partial A_{2}}{\partial s_{1}} M_{22}\right]-T_{13}+\frac{1}{A_{1}} \frac{\partial}{\partial s_{2}}\left(A_{1} M_{21}\right)=\rho \frac{h^{3}}{12} \frac{\partial^{2} \varphi_{1}}{\partial t^{2}} ;$

$\frac{1}{A_{2}}\left[\frac{\partial}{\partial s_{1}}\left(A_{2} M_{12}\right)+\frac{\partial A_{2}}{\partial s_{1}} M_{21}\right]+\frac{1}{A_{1}} \frac{\partial}{\partial s_{2}}\left(A_{1} M_{22}\right)-T_{23}=\rho \frac{h^{3}}{12} \frac{\partial^{2} \varphi_{2}}{\partial t^{2}}$.

The equations for the $j$-th rib:

$$
\begin{aligned}
& \frac{\partial T_{21 j}}{\partial s_{2}}+\left[T_{11}\right]_{j}=\rho_{j} F_{j}\left(\frac{\partial^{2} u_{1}}{\partial t^{2}} \pm h_{c j} \frac{\partial^{2} \varphi_{1}}{\partial t^{2}}\right), \\
& \frac{\partial T_{22 j}}{\partial s_{2}}+k_{2 j} T_{23 j}+[S]_{j}=\rho_{j} F_{j}\left(\frac{\partial^{2} u_{2}}{\partial t^{2}} \pm h_{c j} \frac{\partial^{2} \varphi_{2}}{\partial t^{2}}\right), \\
& \frac{\partial T_{23 j}}{\partial s_{2}}-k_{2 j} T_{22 j}+\left[T_{13}\right]_{j}=\rho_{j} F_{j} \frac{\partial^{2} u_{3}}{\partial t^{2}}, \\
& \frac{\partial M_{21 j}}{\partial s_{2}} \pm h_{c j} \frac{\partial T_{21 j}}{\partial s_{2}}+\left[M_{11}\right]_{j}=\rho_{j} F_{j}\left( \pm h_{c j} \frac{\partial^{2} u_{1}}{\partial t^{2}}+\left(h_{c j}^{2}+\frac{I_{c r j}}{F_{j}}\right) \frac{\partial^{2} \varphi_{1}}{\partial t^{2}}\right), \\
& \frac{\partial M_{22 j}}{\partial s_{2}}-T_{23 j} \pm h_{c j}\left(\frac{\partial T_{22 j}}{\partial s_{2}}+k_{2 j} T_{23 j}\right)+[H]_{j}= \\
& =\rho_{j} F_{j}\left( \pm h_{c j} \frac{\partial^{2} u_{2}}{\partial t^{2}}+\left(h_{c j}^{2}+\frac{I_{2 j}}{F_{j}}\right) \frac{\partial^{2} \varphi_{2}}{\partial t^{2}}\right) .
\end{aligned}
$$

The notation of the type $[S]_{j}$ corresponds to the total effect of the force and moments for the smooth ellipsoidal shell on the $j$-th reinforcing rib in the oscillations equations of the discrete reinforcing ribs (11).
The oscillation equations (10) and (11) are complemented by the corresponding natural boundary and initial conditions.

\section{Numerical algorithm}

The equations (10) and (11) are a system of nonlinear partial differential equations. This system has spatial discontinuities along $s_{2}$. The numerical algorithm is built: a solution is sought in a smooth region of shell and on lines of $j$-th reinforcing rib. We use an integral-interpolation method for spatial coordinates and an explicit approximation in time [15].

The displacement vector is approximated at integer points of the difference grid. The deformation values are approximated at the half-integer points of the grid.

The transition from a continuous to a finite-difference system is performed in two stages. The first stage consists of the finite-difference approximation of the equations of oscillations in the forces-moments.

Operating of integrating equations (10), using an explicit approximation in the time coordinate, we obtain the following difference equations in a smooth region of an ellipsoidal shell:

$$
\begin{aligned}
& \frac{1}{A_{2 l}}\left(\frac{A_{2 l+1 / 2} T_{11 l+1 / 2, m}^{n}-A_{2 l-1 / 2} T_{11 l-1 / 2, m}^{n}}{\Delta s_{1}}\right)-\frac{1}{A_{2 l}} \frac{A_{2 l+1 / 2}-A_{2 l-1 / 2}}{\Delta s_{1}} T_{22 l, m}^{n}+ \\
& \quad+\frac{1}{A_{1 l}} \frac{A_{1 l} T_{21 l, m+1 / 2}^{n}-A_{1 l} T_{21 l, m-1 / 2}^{n}}{\Delta s_{2}}+k_{1 l} T_{13 l, m}^{n}=\rho h\left(u_{1 l, m}^{n}\right)_{\bar{t} t} ;
\end{aligned}
$$

$$
\frac{1}{A_{2 l}}\left(\frac{A_{2 l+1 / 2} T_{12 l+1 / 2, m}^{n}-A_{2 l-1 / 2} T_{12 l-1 / 2, m}^{n}}{\Delta s_{1}}\right)-\frac{1}{A_{2 l}} \frac{A_{2 l+1 / 2}-A_{2 l-1 / 2}}{\Delta s_{1}} T_{21 l, m}^{n}+
$$$$
+\frac{1}{A_{1 l}}\left(\frac{A_{1 l} T_{22 l, m+1 / 2}^{n}-A_{1 l} T_{22 l, m-1 / 2}^{n}}{\Delta s_{2}}\right)+k_{2 l} T_{23 l, m}^{n}=\rho h\left(u_{2 l, m}^{n}\right)_{\overline{i t}} ;
$$$$
\frac{1}{A_{2 l}}\left(\frac{A_{2 l+1 / 2} T_{13 l+1 / 2, m}^{n}-A_{2 l-1 / 2} T_{13 l-1 / 2, m}^{n}}{\Delta s_{1}}\right)-k_{1 l} T_{11 l, m}^{n}+
$$$$
+\frac{1}{A_{1 l}}\left(\frac{A_{1 l} T_{23 l, m+1 / 2}^{n}-A_{1 l} T_{23 l, m-1 / 2}^{n}}{\Delta s_{2}}\right)-k_{2 l} T_{22 l, m}^{n}+P_{3 l, m}^{n}=\rho h\left(u_{3 l, m}^{n}\right)_{i t} ;
$$$$
\frac{1}{A_{2 l}}\left(\frac{A_{2 l+1 / 2} M_{11 l+1 / 2, m}^{n}-A_{2 l-1 / 2} M_{11 l-1 / 2, m}^{n}}{\Delta s_{1}}\right)-\frac{1}{A_{2 l}} \frac{A_{2 l+1 / 2}-A_{2 l-1 / 2}}{\Delta s_{1}} M_{22 l, m}^{n}+
$$$$
+\frac{1}{A_{1 l}}\left(\frac{A_{1 l} M_{21 l, m+1 / 2}^{n}-A_{1 l} M_{21 l, m-1 / 2}^{n}}{\Delta s_{2}}\right)-T_{13 l, m}^{n}=\frac{\rho h^{3}}{12}\left(\varphi_{1 l, m}^{n}\right)_{\bar{t} t}
$$

$$
\begin{gathered}
\frac{1}{A_{2 l}}\left(\frac{A_{2 l+1 / 2} M_{12 l+1 / 2, m}^{n}-A_{2 l-1 / 2} M_{12 l-1 / 2, m}^{n}}{\Delta s_{1}}\right)-\frac{1}{A_{2 l}} \frac{A_{2 l+1 / 2}-A_{2 l-1 / 2}}{\Delta s_{1}} M_{21 l, m}^{n}+ \\
+\frac{1}{A_{1 l}}\left(\frac{A_{1 l} M_{22 l, m+1 / 2}^{n}-A_{1 l} M_{22 l, m-1 / 2}^{n}}{\Delta s_{2}}\right)-T_{23 l, m}^{n}=\frac{\rho h^{3}}{12}\left(\varphi_{2 l, m}^{n}\right)_{\bar{t} t}
\end{gathered}
$$


In difference equations (12), the components of the generalized displacements vector of the middle surface for the smooth ellipsoidal shell $\bar{U}=\left(u_{1}, u_{2}, u_{3}, \varphi_{1}, \varphi_{2}\right)^{T}$ are assigned to whole nodes of the different grid $\bar{U}_{l, m}=\left(u_{1 l, m}, u_{2 l, m}, u_{3 l, m}, \varphi_{1 l, m}, \varphi_{2 l, m}\right)^{T}$ in spatial coordinates.

Operating of integrating equations (11), using an explicit approximation in the time coordinate, we obtain the following difference equations for $j$-th reinforcing rib:

$$
\begin{aligned}
& \frac{T_{21 j m+1 / 2}^{n}-T_{21 j m-1 / 2}^{n}}{\Delta s_{2}}+\left[T_{11}\right]_{j}^{n}=\rho_{j} F_{j}\left[\left(u_{1 m}^{n}\right)_{t t} \pm h_{c i}\left(\varphi_{1 m}^{n}\right)_{t t}\right] \\
& \frac{T_{22 j m+1 / 2}^{n}-T_{22 j m-1 / 2}^{n}}{\Delta S_{2}}+k_{2 j m} T_{23 j m}^{n}+[S]_{j}^{n}= \\
& =\rho_{j} F_{j}\left[\left(u_{2 m}^{n}\right)_{i t} \pm h_{c i}\left(\varphi_{2 m}^{n}\right)_{i t}\right\rfloor \text {, } \\
& \frac{T_{23 j m+1 / 2}^{n}-T_{23 j m-1 / 2}^{n}}{\Delta S_{2}}-k_{2 j m} T_{22 j m}^{n}+\left[T_{13}\right]_{j}^{n}=\rho_{j} F_{j}\left(u_{3 m}^{n}\right)_{t t}, \\
& \frac{M_{21 j m+1 / 2}^{n}-M_{21 j m-1 / 2}^{n}}{\Delta s_{2}} \pm h_{c j} \frac{T_{21 j m+1 / 2}^{n}-T_{21 j m-1 / 2}^{n}}{\Delta s_{2}}+\left[M_{11}\right]_{j}^{n}= \\
& =\rho_{j} F_{j}\left[ \pm h_{c j}\left(u_{1 m}^{n}\right)_{t t}+\left(h_{c j}^{2}+\frac{I_{c r j}}{F_{j}}\right)\left(\varphi_{1 m}^{n}\right)_{t t}\right] \text {, } \\
& \frac{M_{22 j m+1 / 2}^{n}-M_{22 j m-1 / 2}^{n}}{\Delta s_{2}}-T_{23 j m}^{n} \pm h_{c j}\left(\frac{T_{22 j m+1 / 2}^{n}-T_{22 j m-1 / 2}^{n}}{\Delta s_{2}}+k_{2 j m} T_{23 j m}^{n}\right)+ \\
& +[H]_{j}^{n}=\rho_{j} F_{j}\left[ \pm h_{c j}\left(u_{2 m}^{n}\right)_{i t}+\left(h_{c j}^{2}+\frac{I_{2 j}}{F_{j}}\right)\left(\varphi_{2 m}^{n}\right)_{i t}\right] \text {. }
\end{aligned}
$$

The components of the generalized displacements vector of the mass centers in the cross-sections for the $j$-th reinforcing $\mathrm{rib} \bar{U}_{j}=\left(u_{1 j}, u_{2 j}, u_{3 j}, \varphi_{1 j}, \varphi_{2 j}\right)^{T}$ are assigned to integer nodes of the difference mesh in spatial coordinates.

In the second stage of approximation, the equations consist of finite-difference approximation of forces-moments and corresponding deformations.

We use the necessary conditions of stability

$$
\Delta t \leq 2 \text { / } \omega
$$

where $\omega=\max \left(\omega_{0}, \omega_{j}\right), j=1, J ; \omega_{0}, \omega_{j}-$ frequency of natural oscillations the discrete-difference system for the smooth shell and the ${ }^{j}$-th reinforcing rib.

\section{Numerical example}

We investigated the change in deformation for discretely reinforced ellipsoidal in the region:

$$
D=\left\{\alpha_{10} \leq \alpha_{1} \leq \alpha_{1 N}, \alpha_{20} \leq \alpha_{2} \leq \alpha_{2 N}\right\} .
$$

The load on the shell $P_{3}\left(\alpha_{1}, \alpha_{2}, t\right)$ has the form:

$$
\begin{gathered}
P_{3}\left(\alpha_{1}, \alpha_{2}, t\right)=A \cdot \sin \frac{\pi t}{T}[\eta(t)-\eta(t-T)], \\
A=10^{6} \Pi a ; \quad T=50 \cdot 10^{-6} c .
\end{gathered}
$$

The boundary conditions are as follows

$\bar{U}\left(\alpha_{10}, \alpha_{2}\right)=\bar{U}\left(\alpha_{1 N}, \alpha_{2}\right)=0 ; \quad \bar{U}\left(\alpha_{1}, \alpha_{20}\right)=\bar{U}\left(\alpha_{1}, \alpha_{2 N}\right)=0$.
The generalized displacement vector has zero components at $t=0$.

The geometrical parameters of the shell and rib:

$$
\begin{aligned}
& \alpha_{10}=\frac{\pi}{12} ; \quad \alpha_{1 N}=\pi-\frac{\pi}{12} ; \quad \alpha_{20}=-\frac{\pi}{2} ; \quad \alpha_{2 N}=\frac{\pi}{2} ; \\
& \frac{b}{a}=1,5 ; \quad \frac{a}{h}=30 ; \\
& h_{j}=4 \cdot h ; \quad F_{j}=4 \cdot h^{2} .
\end{aligned}
$$

The physical and mechanical parameters:

$$
\begin{array}{cll}
E=7 \cdot 10^{10} \Pi a ; & v=0,33 ; & \rho=2,7 \cdot 10^{3} \kappa 2 / \mathcal{M}^{3} ; \\
E_{j}=E_{1} ; & \rho_{j}=\rho .
\end{array}
$$

The location of the reinforcing ribs was investigated for the following 5 cases.

1. External placement of ribs on the shell (curve 1)

$$
\alpha_{1 j}=\frac{\pi}{4} j, j=\overline{1,3} \text {. }
$$

2. Internal placement of ribs in the shell (curve 2)

$$
\alpha_{1 j}=\frac{\pi}{8}+\frac{\pi}{4} j, j=\overline{0,3} \text {. }
$$

3. External placement of all ribs on the shell (curve 3)

$$
\alpha_{1 j}=\frac{\pi}{8}+\frac{\pi}{8} j, j=\overline{0,6} \text {. }
$$

4. Internal placement of all ribs in the shell (curve 4)

$$
\alpha_{1 j}=\frac{\pi}{8}+\frac{\pi}{8} j, j=\overline{0,6} \text {. }
$$

5. External placement of ribs $\alpha_{1 j}=\frac{\pi}{4} j(j=\overline{1,3})$ on the shell and internal placement of ribs $\alpha_{1 j}=\frac{\pi}{8}+\frac{\pi}{4} j$ $(j=\overline{0,3})$ in the shell. This is a curve 5 .

Calculations were performed on the time interval $t_{N}=20 T$. Fig. $1-9$ show the deformations $\varepsilon_{22}$, that make it possible to analyze the deformed state of the reinforced ellipsoidal shell.

We investigated the change in the amount of deformation at the most characteristic points of the reinforced shell: at the points of ribs placement and between the ribs.

Fig. 1 shows the changes of deformations $\varepsilon_{22}$ at the point $\left(\alpha_{1}=\frac{\pi}{8}, \alpha_{2}=0\right)$, where the rib is placed. 


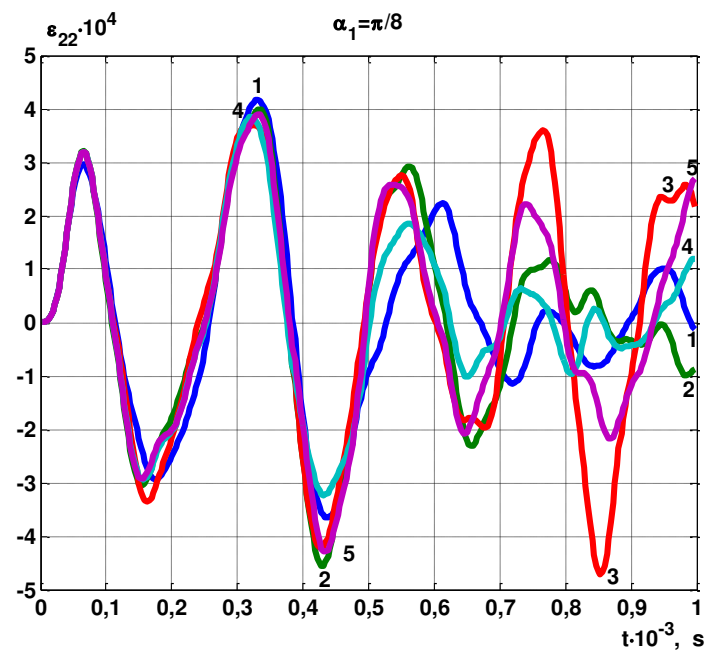

Fig. 1 Deformations $\varepsilon_{22}\left(\frac{\pi}{8} ; 0\right)$ on time $t$

The largest absolute values of deformations $\varepsilon_{22}\left(\frac{\pi}{8} ; 0\right)$ for given cases:

$$
\begin{aligned}
& \varepsilon_{22}^{1}\left(\frac{\pi}{8} ; 0\right)= 4,17 \cdot 10^{-4} ; \quad \varepsilon_{22}^{2}\left(\frac{\pi}{8} ; 0\right)=4,56 \cdot 10^{-4} ; \\
& \varepsilon_{22}^{3}\left(\frac{\pi}{8} ; 0\right)=4,72 \cdot 10^{-4} ; \\
& \varepsilon_{22}^{4}\left(\frac{\pi}{8} ; 0\right)=3,86 \cdot 10^{-4} ; \quad \varepsilon_{22}^{5}\left(\frac{\pi}{8} ; 0\right)=4,29 \cdot 10^{-4} .
\end{aligned}
$$

The value of deformation $\varepsilon_{22}^{4}\left(\frac{\pi}{8} ; 0\right)$ is the smallest, and $\varepsilon_{22}^{3}\left(\frac{\pi}{8} ; 0\right)$ - the largest, which is 1,2 times more:

$$
\frac{\varepsilon_{22}^{3}\left(\frac{\pi}{8} ; 0\right)}{\varepsilon_{22}^{4}\left(\frac{\pi}{8} ; 0\right)}=\frac{4,72 \cdot 10^{-4}}{3,86 \cdot 10^{-4}} \approx 1,2
$$

The average value of the achieved deformations $\varepsilon_{22}$ at the point $\left(\alpha_{1}=\frac{\pi}{8}, \alpha_{2}=0\right)$ is equal to

$$
\bar{\varepsilon}_{22}\left(\frac{\pi}{8} ; 0\right)=\frac{1}{5} \sum_{n=1}^{5} \varepsilon_{22}^{n}\left(\frac{\pi}{8} ; 0\right)=4,32 \cdot 10^{-4} .
$$

The changes of deformations $\varepsilon_{22}$ between the ribs at the point $\left(\alpha_{1}=\frac{3 \pi}{16}, \alpha_{2}=0\right)$ are shown in Fig. 2

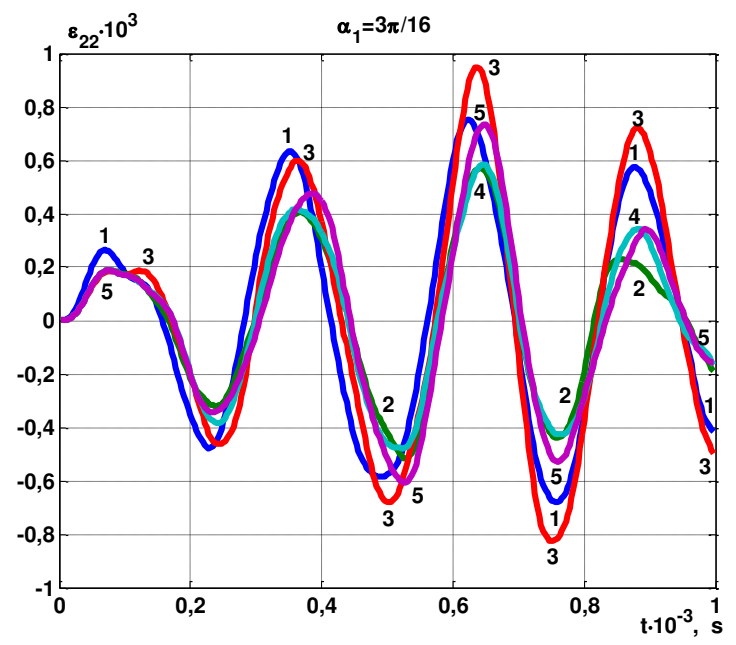

Fig. 2 Deformations $\varepsilon_{22}\left(\frac{3 \pi}{16} ; 0\right)$ on time $t$

The largest absolute values of deformations $\varepsilon_{22}\left(\frac{3 \pi}{16} ; 0\right)$ for given cases:

$$
\begin{gathered}
\varepsilon_{22}^{1}\left(\frac{3 \pi}{16} ; 0\right)=7,53 \cdot 10^{-4} ; \quad \varepsilon_{22}^{2}\left(\frac{3 \pi}{16} ; 0\right)=5,70 \cdot 10^{-4} ; \\
\varepsilon_{22}^{3}\left(\frac{3 \pi}{16} ; 0\right)=9,50 \cdot 10^{-4} ; \\
\varepsilon_{22}^{4}\left(\frac{3 \pi}{16} ; 0\right)=5,86 \cdot 10^{-4} ; \quad \varepsilon_{22}^{5}\left(\frac{3 \pi}{16} ; 0\right)=7,36 \cdot 10^{-4} .
\end{gathered}
$$

The value of deformation $\varepsilon_{22}^{2}\left(\frac{3 \pi}{16} ; 0\right)$ is the smallest, and $\varepsilon_{22}^{3}\left(\frac{3 \pi}{16} ; 0\right)$ - the largest, which is 1,7 times more:

$$
\frac{\varepsilon_{22}^{3}\left(\frac{3 \pi}{16} ; 0\right)}{\varepsilon_{22}^{2}\left(\frac{3 \pi}{16} ; 0\right)}=\frac{9,50 \cdot 10^{-4}}{5,70 \cdot 10^{-4}} \approx 1,7
$$

The average value of deformations $\varepsilon_{22}$ at the point $\left(\alpha_{1}=\frac{3 \pi}{16}, \alpha_{2}=0\right)$ is equal to

$$
\bar{\varepsilon}_{22}\left(\frac{3 \pi}{16} ; 0\right)=\frac{1}{5} \sum_{n=1}^{5} \varepsilon_{22}^{n}\left(\frac{3 \pi}{16} ; 0\right)=7,19 \cdot 10^{-4} .
$$

Fig. 3 represents the changes of deformations $\varepsilon_{22}$ at the point $\left(\alpha_{1}=\frac{\pi}{4}, \alpha_{2}=0\right)$, where the rib is placed. 


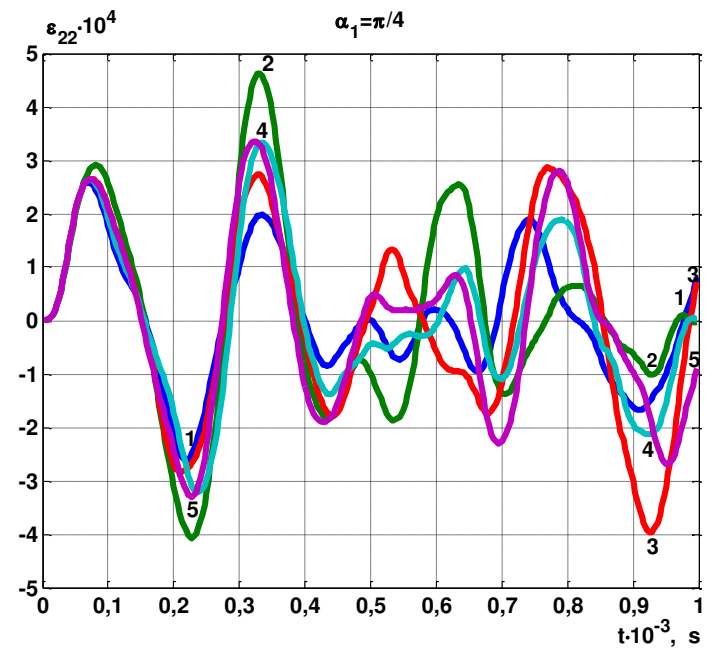

Fig. 3 Deformations $\varepsilon_{22}\left(\frac{\pi}{4} ; 0\right)$ on time $t$

The largest absolute values of deformations $\varepsilon_{22}\left(\frac{\pi}{4} ; 0\right)$ for given cases:

$$
\begin{aligned}
& \varepsilon_{22}^{1}\left(\frac{\pi}{4} ; 0\right)=2,61 \cdot 10^{-4} ; \quad \varepsilon_{22}^{2}\left(\frac{\pi}{4} ; 0\right)=4,63 \cdot 10^{-4} ; \\
& \varepsilon_{22}^{3}\left(\frac{\pi}{4} ; 0\right)=3,96 \cdot 10^{-4} ; \\
& \varepsilon_{22}^{4}\left(\frac{\pi}{4} ; 0\right)=3,34 \cdot 10^{-4} ; \quad \varepsilon_{22}^{5}\left(\frac{\pi}{4} ; 0\right)=3,35 \cdot 10^{-4} .
\end{aligned}
$$

After analyzing the value of deformation $\varepsilon_{22}$ we can conclude that $\varepsilon_{22}^{1}\left(\frac{\pi}{4} ; 0\right)$ is the smallest, and $\varepsilon_{22}^{1}\left(\frac{\pi}{4} ; 0\right)$ - the largest, which is 1,8 times more:

$$
\frac{\varepsilon_{22}^{2}\left(\frac{\pi}{4} ; 0\right)}{\varepsilon_{22}^{1}\left(\frac{\pi}{4} ; 0\right)}=\frac{4,63 \cdot 10^{-4}}{2,61 \cdot 10^{-4}} \approx 1,8
$$

After calculating the average value of deformation ${ }^{\varepsilon_{22}}$ at the point $\left(\alpha_{1}=\frac{\pi}{4}, \alpha_{2}=0\right)$ we receive:

$$
\bar{\varepsilon}_{22}\left(\frac{\pi}{4} ; 0\right)=\frac{1}{5} \sum_{n=1}^{5} \varepsilon_{22}^{n}\left(\frac{\pi}{4} ; 0\right)=3,58 \cdot 10^{-4} .
$$

Graphically, the results of calculations for deformations $\varepsilon_{22}$ between the ribs at the point $\left(\alpha_{1}=\frac{5 \pi}{16}, \alpha_{2}=0\right)$ are presented in Fig. 4

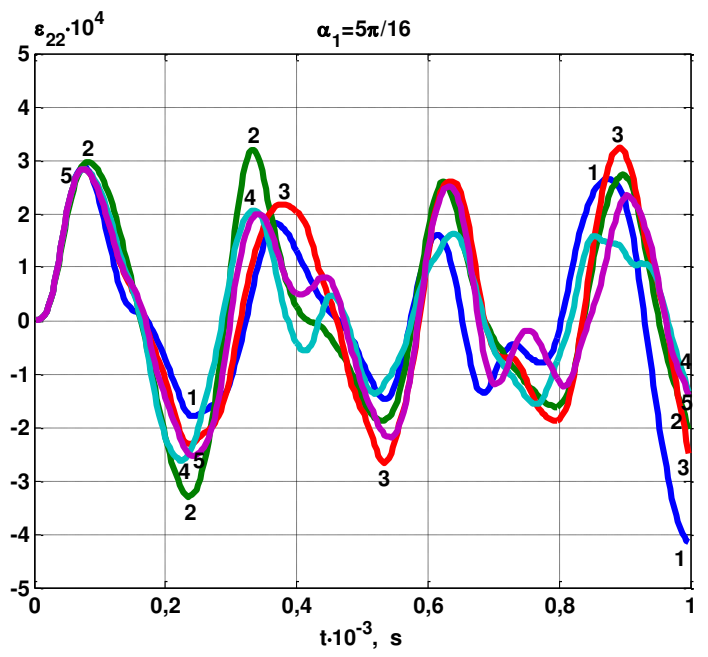

Fig. 4 Deformations $\varepsilon_{22}\left(\frac{5 \pi}{16} ; 0\right)$ on time $t$

The largest absolute values of deformations $\varepsilon_{22}\left(\frac{5 \pi}{16} ; 0\right)$ for given cases:

$$
\begin{aligned}
& \varepsilon_{22}^{1}\left(\frac{5 \pi}{16} ; 0\right)=4,15 \cdot 10^{-4} ; \varepsilon_{22}^{2}\left(\frac{5 \pi}{16} ; 0\right)=3,30 \cdot 10^{-4} ; \\
& \varepsilon_{22}^{3}\left(\frac{5 \pi}{16} ; 0\right)=3,23 \cdot 10^{-4} ; \\
& \varepsilon_{22}^{4}\left(\frac{5 \pi}{16} ; 0\right)=2,84 \cdot 10^{-4} ; \quad \varepsilon_{22}^{5}\left(\frac{5 \pi}{16} ; 0\right)=2,84 \cdot 10^{-4} .
\end{aligned}
$$

After analyzing the results, we can conclude that the value of deformation $\varepsilon_{22}^{4}\left(\frac{5 \pi}{16} ; 0\right)$ or $\varepsilon_{22}^{5}\left(\frac{5 \pi}{16} ; 0\right)$ is the smallest, and $\varepsilon_{22}^{1}\left(\frac{5 \pi}{16} ; 0\right)$ - the largest, which is 1,5 times more:

$$
\frac{\varepsilon_{22}^{1}\left(\frac{5 \pi}{16} ; 0\right)}{\varepsilon_{22}^{4}\left(\frac{5 \pi}{16} ; 0\right)}=\frac{4,15 \cdot 10^{-4}}{2,84 \cdot 10^{-4}} \approx 1,5
$$

Using the found values of deformation $\varepsilon_{22}$ at the point $\left(\alpha_{1}=\frac{5 \pi}{16}, \alpha_{2}=0\right)$ we calculate the average value of deformation at this point:

$$
\bar{\varepsilon}_{22}\left(\frac{5 \pi}{16} ; 0\right)=\frac{1}{5} \sum_{n=1}^{5} \varepsilon_{22}^{n}\left(\frac{5 \pi}{16} ; 0\right)=3,27 \cdot 10^{-4} .
$$

The results of deformation calculations $\varepsilon_{22}$ at the point $\left(\alpha_{1}=\frac{3 \pi}{8}, \alpha_{2}=0\right)$ in the rib are shown in Figure 5 . 


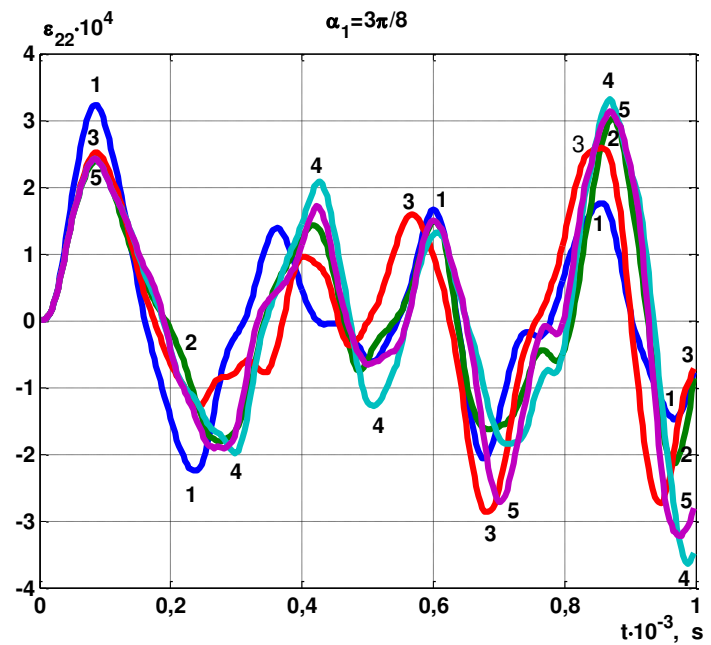

Fig. 5 Deformations $\varepsilon_{22}\left(\frac{3 \pi}{8} ; 0\right)$ on time $t$

The largest absolute values of deformations $\varepsilon_{22}\left(\frac{3 \pi}{8} ; 0\right)$ for given cases:

$$
\begin{gathered}
\varepsilon_{22}^{1}\left(\frac{3 \pi}{8} ; 0\right)=3,23 \cdot 10^{-4} ; \quad \varepsilon_{22}^{2}\left(\frac{3 \pi}{8} ; 0\right)=3,03 \cdot 10^{-4} ; \\
\varepsilon_{22}^{3}\left(\frac{3 \pi}{8} ; 0\right)=2,87 \cdot 10^{-4} ; \\
\varepsilon_{22}^{4}\left(\frac{3 \pi}{8} ; 0\right)=3,64 \cdot 10^{-4} ; \quad \varepsilon_{22}^{5}\left(\frac{3 \pi}{8} ; 0\right)=3,22 \cdot 10^{-4} .
\end{gathered}
$$

Analyzing the value of deformations at the point $\left(\alpha_{1}=\frac{3 \pi}{8}, \alpha_{2}=0\right)$, we can say that the value of deformation $\varepsilon_{22}^{3}\left(\frac{3 \pi}{8} ; 0\right)$ is the smallest, and $\varepsilon_{22}^{4}\left(\frac{3 \pi}{8} ; 0\right)$ - the largest, which is 1,3 times more:

$$
\frac{\varepsilon_{22}^{4}\left(\frac{3 \pi}{8} ; 0\right)}{\varepsilon_{22}^{3}\left(\frac{3 \pi}{8} ; 0\right)}=\frac{3,64 \cdot 10^{-4}}{2,87 \cdot 10^{-4}} \approx 1,3
$$

At the point $\left(\alpha_{1}=\frac{3 \pi}{8}, \alpha_{2}=0\right)$ the average value of deformations $\varepsilon_{22}$ is equal to

$$
\bar{\varepsilon}_{22}\left(\frac{3 \pi}{8} ; 0\right)=\frac{1}{5} \sum_{n=1}^{5} \varepsilon_{22}^{n}\left(\frac{3 \pi}{8} ; 0\right)=3,2 \cdot 10^{-4} .
$$

The changes of deformations $\varepsilon_{22}$ between the ribs at the point $\left(\alpha_{1}=\frac{7 \pi}{16}, \alpha_{2}=0\right)$ are shown in Fig. 6 .

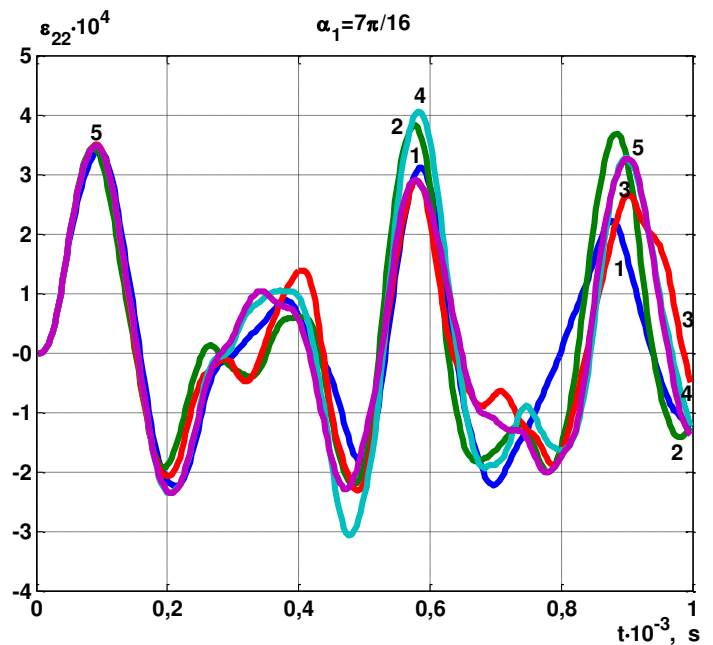

Fig. 6 Deformations $\varepsilon_{22}\left(\frac{7 \pi}{16} ; 0\right)$ on time $t$

The largest absolute values of deformations $\varepsilon_{22}\left(\frac{7 \pi}{16} ; 0\right)$ for given cases:

$$
\begin{gathered}
\varepsilon_{22}^{1}\left(\frac{7 \pi}{16} ; 0\right)=3,43 \cdot 10^{-4} ; \quad \varepsilon_{22}^{2}\left(\frac{7 \pi}{16} ; 0\right)=3,84 \cdot 10^{-4} ; \\
\varepsilon_{22}^{3}\left(\frac{7 \pi}{16} ; 0\right)=3,51 \cdot 10^{-4} ; \\
\varepsilon_{22}^{4}\left(\frac{7 \pi}{16} ; 0\right)=4,07 \cdot 10^{-4} ; \quad \varepsilon_{22}^{5}\left(\frac{7 \pi}{16} ; 0\right)=3,49 \cdot 10^{-4} .
\end{gathered}
$$

We can conclude that the value of deformation $\varepsilon_{22}^{1}\left(\frac{7 \pi}{16} ; 0\right)$ is the smallest, and $\varepsilon_{22}^{4}\left(\frac{7 \pi}{16} ; 0\right)$ - the largest, which is 1,2 times more:

$$
\frac{\varepsilon_{22}^{4}\left(\frac{7 \pi}{16} ; 0\right)}{\varepsilon_{22}^{1}\left(\frac{7 \pi}{16} ; 0\right)}=\frac{4,07 \cdot 10^{-4}}{3,43 \cdot 10^{-4}} \approx 1,2
$$

The average value of deformations $\varepsilon_{22}$ at the point $\left(\alpha_{1}=\frac{7 \pi}{16}, \alpha_{2}=0\right)$ is equal to

$$
\bar{\varepsilon}_{22}\left(\frac{7 \pi}{16} ; 0\right)=\frac{1}{5} \sum_{n=1}^{5} \varepsilon_{22}^{n}\left(\frac{7 \pi}{16} ; 0\right)=3,67 \cdot 10^{-4} .
$$

Fig. 7 shows the changes of deformation $\varepsilon_{22}$ at the point $\left(\alpha_{1}=\frac{\pi}{2}, \alpha_{2}=0\right)$ in the rib. 


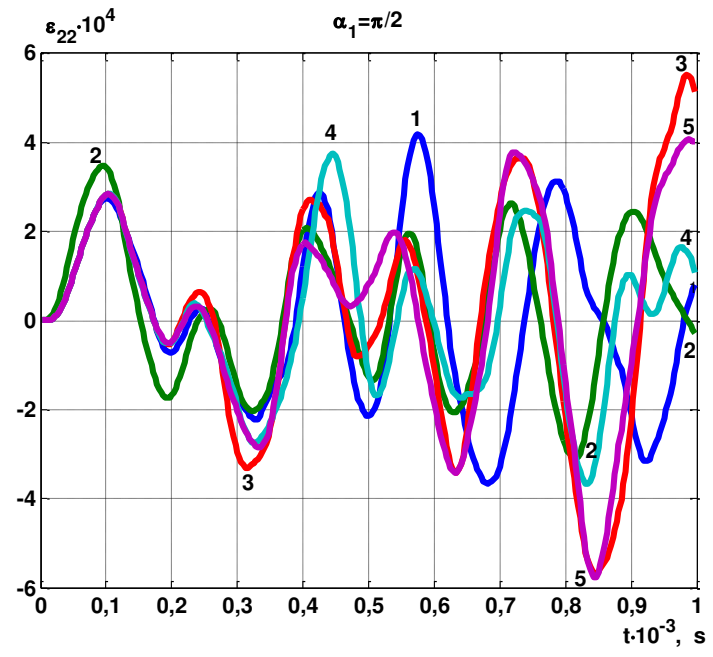

Fig. 7 Deformations $\varepsilon_{22}\left(\frac{\pi}{2} ; 0\right)$ on time $t$

The largest absolute values of deformations $\varepsilon_{22}\left(\frac{\pi}{2} ; 0\right)$ for given cases:

$$
\begin{aligned}
& \varepsilon_{22}^{1}\left(\frac{\pi}{2} ; 0\right)=4,18 \cdot 10^{-4} ; \quad \varepsilon_{22}^{2}\left(\frac{\pi}{2} ; 0\right)=3,47 \cdot 10^{-4} ; \\
& \varepsilon_{22}^{3}\left(\frac{\pi}{2} ; 0\right)=5,71 \cdot 10^{-4} ; \\
& \varepsilon_{22}^{4}\left(\frac{\pi}{2} ; 0\right)=3,75 \cdot 10^{-4} ; \quad \varepsilon_{22}^{5}\left(\frac{\pi}{2} ; 0\right)=5,76 \cdot 10^{-4} .
\end{aligned}
$$

After analyzing the results, we can say that the value of deformation $\varepsilon_{22}^{2}\left(\frac{\pi}{2} ; 0\right)$ is the smallest, and $\varepsilon_{22}^{5}\left(\frac{\pi}{2} ; 0\right)$ - the largest, which is 1,7 times more:

$$
\frac{\varepsilon_{22}^{5}\left(\frac{\pi}{2} ; 0\right)}{\varepsilon_{22}^{2}\left(\frac{\pi}{2} ; 0\right)}=\frac{5,76 \cdot 10^{-4}}{3,47 \cdot 10^{-4}} \approx 1,7
$$

We calculate the average value of deformations $\varepsilon_{22}$ at the point $\left(\alpha_{1}=\frac{\pi}{2}, \alpha_{2}=0\right)$ :

$$
\bar{\varepsilon}_{22}\left(\frac{\pi}{2} ; 0\right)=\frac{1}{5} \sum_{n=1}^{5} \varepsilon_{22}^{n}\left(\frac{\pi}{2} ; 0\right)=4,57 \cdot 10^{-4} .
$$

Therefore, the largest difference for deformations $\varepsilon_{22}$ is manifested for cases of external and internal placement of ribs (cases 1 and 2 ) at the point $\left(\alpha_{1}=\frac{\pi}{4}, \alpha_{2}=0\right)$ :

$$
\frac{\varepsilon_{22}^{2}\left(\frac{\pi}{4} ; 0\right)}{\varepsilon_{22}^{1}\left(\frac{\pi}{4} ; 0\right)} \approx 1,8
$$

After analyzing the results for case 1, we can conclude that the largest value of deformation between the ribs $\varepsilon_{22}^{1}\left(\frac{3 \pi}{16} ; 0\right)$ is 1,8 times more than the largest value of deformation in the rib $\varepsilon_{22}^{1}\left(\frac{\pi}{2} ; 0\right)$ :

$$
\frac{\varepsilon_{22}^{1}\left(\frac{3 \pi}{16} ; 0\right)}{\varepsilon_{22}^{1}\left(\frac{\pi}{2} ; 0\right)}=\frac{7,53 \cdot 10^{-4}}{4,18 \cdot 10^{-4}} \approx 1,8
$$

Considering the results for case 2 , we can say that the largest value of deformation between the ribs $\varepsilon_{22}^{2}\left(\frac{3 \pi}{16} ; 0\right)$ is 1,2 times more than the largest value of deformation in the rib $\varepsilon_{22}^{2}\left(\frac{\pi}{4} ; 0\right)$ :

$$
\frac{\varepsilon_{22}^{2}\left(\frac{3 \pi}{16} ; 0\right)}{\varepsilon_{22}^{2}\left(\frac{\pi}{4} ; 0\right)}=\frac{5,70 \cdot 10^{-4}}{4,63 \cdot 10^{-4}} \approx 1,2
$$

Discussing the results for case 3 , we can conclude that the largest value of deformation between the ribs $\varepsilon_{22}^{3}\left(\frac{3 \pi}{16} ; 0\right)$ is 1,7 times more than the largest value of deformation in the rib $\varepsilon_{22}^{3}\left(\frac{\pi}{2} ; 0\right)$ :

$$
\frac{\varepsilon_{22}^{3}\left(\frac{3 \pi}{16} ; 0\right)}{\varepsilon_{22}^{3}\left(\frac{\pi}{2} ; 0\right)}=\frac{9,50 \cdot 10^{-4}}{5,71 \cdot 10^{-4}} \approx 1,7
$$

After analyzing the results for case 4, we can assume that the largest value of deformation between the ribs $\varepsilon_{22}^{4}\left(\frac{3 \pi}{16} ; 0\right)$ is 1,5 times more than the largest value of deformation in the rib $\varepsilon_{22}^{4}\left(\frac{\pi}{8} ; 0\right)$ :

$$
\frac{\varepsilon_{22}^{4}\left(\frac{3 \pi}{16} ; 0\right)}{\varepsilon_{22}^{4}\left(\frac{\pi}{8} ; 0\right)}=\frac{5,86 \cdot 10^{-4}}{3,86 \cdot 10^{-4}} \approx 1,5
$$

According to results for case 5, we can conclude that the largest value of deformation between the ribs $\varepsilon_{22}^{5}\left(\frac{3 \pi}{16} ; 0\right)$ is 1,3 times more than the largest value of deformation in the rib $\varepsilon_{22}^{5}\left(\frac{\pi}{2} ; 0\right)$ :

$$
\frac{\varepsilon_{22}^{5}\left(\frac{3 \pi}{16} ; 0\right)}{\varepsilon_{22}^{5}\left(\frac{\pi}{2} ; 0\right)}=\frac{7,36 \cdot 10^{-4}}{5,76 \cdot 10^{-4}} \approx 1,3
$$

Based on the above, we conclude:

1) the largest values of deformations between the rib are $\varepsilon_{22}^{3}\left(\frac{3 \pi}{16} ; 0\right)=9,5 \cdot 10^{-4} ;$

2) the largest value of deformation in the rib is 


$$
\varepsilon_{22}^{5}\left(\frac{\pi}{2} ; 0\right)=5,76 \cdot 10^{-4} ;
$$

3) the smallest value of deformation between the rib is

$$
\varepsilon_{22}^{5}\left(\frac{5 \pi}{16} ; 0\right)=2,84 \cdot 10^{-4} ;
$$

4) the smallest value of deformation in the rib is

$$
\varepsilon_{22}^{1}\left(\frac{\pi}{4} ; 0\right)=2,61 \cdot 10^{-4} \text {. }
$$

Let's carry out the comparative analysis of deformations $\varepsilon_{22}$ for the considered cases of the ribs locations.

The average values of deformations $\varepsilon_{22}$ for case 1 are equal to

$\bar{\varepsilon}_{22}^{1}\left(\frac{\pi}{8} ; \frac{\pi}{4} ; \frac{3 \pi}{8} ; \frac{\pi}{2}\right)=3,75 \cdot 10^{-4}$ (in the ribs),

$\bar{\varepsilon}_{22}^{1}\left(\frac{3 \pi}{16} ; \frac{5 \pi}{16} ; \frac{7 \pi}{16}\right)=5,04 \cdot 10^{-4}$ (between the ribs).

After calculating the average values of deformations $\varepsilon_{22}$ for case 2 we receive

$\bar{\varepsilon}_{22}^{2}\left(\frac{\pi}{8} ; \frac{\pi}{4} ; \frac{3 \pi}{8} ; \frac{\pi}{2}\right)=3,92 \cdot 10^{-4}$ (in the ribs),

$\bar{\varepsilon}_{22}^{2}\left(\frac{3 \pi}{16} ; \frac{5 \pi}{16} ; \frac{7 \pi}{16}\right)=4,28 \cdot 10^{-4}$ (between the ribs).

Using the found values of deformation $\varepsilon_{22}$ for case 3 we calculate the average values of deformations

$\bar{\varepsilon}_{22}^{3}\left(\frac{\pi}{8} ; \frac{\pi}{4} ; \frac{3 \pi}{8} ; \frac{\pi}{2}\right)=4,32 \cdot 10^{-4}$ (in the ribs),

$\bar{\varepsilon}_{22}^{3}\left(\frac{3 \pi}{16} ; \frac{5 \pi}{16} ; \frac{7 \pi}{16}\right)=5,41 \cdot 10^{-4}$ (between the ribs).

For case 4 the average values of deformations $\varepsilon_{22}$ are equal to

$$
\begin{aligned}
& \bar{\varepsilon}_{22}^{4}\left(\frac{\pi}{8} ; \frac{\pi}{4} ; \frac{3 \pi}{8} ; \frac{\pi}{2}\right)=3,65 \cdot 10^{-4} \text { (in the ribs), } \\
& \bar{\varepsilon}_{22}^{4}\left(\frac{3 \pi}{16} ; \frac{5 \pi}{16} ; \frac{7 \pi}{16}\right)=4,26 \cdot 10^{-4} \text { (between the ribs). }
\end{aligned}
$$

We can conclude that the average values of deformations $\varepsilon_{22}$ for case 5 are equal to

$$
\begin{aligned}
& \bar{\varepsilon}_{22}^{5}\left(\frac{\pi}{8} ; \frac{\pi}{4} ; \frac{3 \pi}{8} ; \frac{\pi}{2}\right)=4,16 \cdot 10^{-4} \text { (in the ribs), } \\
& \bar{\varepsilon}_{22}^{5}\left(\frac{3 \pi}{16} ; \frac{5 \pi}{16} ; \frac{7 \pi}{16}\right)=4,56 \cdot 10^{-4} \text { (between the ribs). }
\end{aligned}
$$

Therefore, analyzing the average values found, we can say the following. In both cases: at the location of the rib and between the ribs, the largest values of deformations for case 3 are when all the ribs are placed external the ellipsoidal shell. The smallest deformations for case 4, when all the ribs are placed internally in the shell.

Due to the symmetry along the coordinate $\alpha_{1}$, the dependencies are given in the range $\alpha_{10} \leq \alpha_{1} \leq \frac{\pi}{2}$ in the section $\alpha_{2}=0$.

Fig. 8 shows the deformations $\varepsilon_{22}$ at the largest time $t=20 T$.

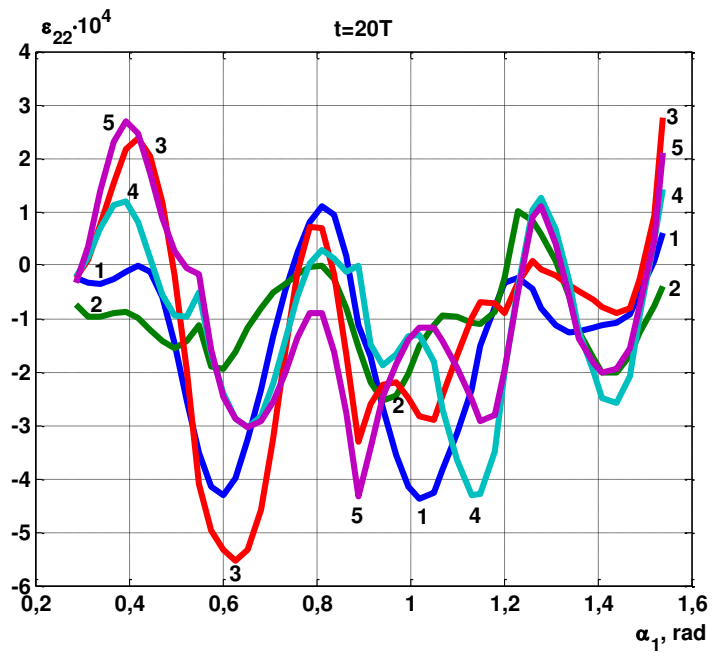

Fig. 8 Deformations $\varepsilon_{22}$ on spatial coordinate $\alpha_{1}$ in crosssection $\alpha_{2}=0$ the time $t=20 T$

The largest absolute values of the deformations $\varepsilon_{22}$ for given cases the time $t=20 T$ :

$$
\begin{aligned}
& \left.\varepsilon_{22}^{1}\left(\frac{5 \pi}{16} ; 0\right)\right|_{t=20 T}=4,37 \cdot 10^{-4} ;\left.\quad \varepsilon_{22}^{2}\left(\frac{5 \pi}{16} ; 0\right)\right|_{t=20 T}=2,54 \cdot 10^{-4} ; \\
& \left.\quad \varepsilon_{22}^{3}\left(\frac{3 \pi}{16} ; 0\right)\right|_{t=20 T}=5,53 \cdot 10^{-4} ; \\
& \left.\varepsilon_{22}^{4}\left(\frac{3 \pi}{8} ; 0\right)\right|_{t=20 T}=4,3 \cdot 10^{-4} ;\left.\quad \varepsilon_{22}^{5}\left(\frac{5 \pi}{16} ; 0\right)\right|_{t=20 T}=4,33 \cdot 10^{-4} .
\end{aligned}
$$

The largest value of deformation at time $t=20 T$ is obtained between the rib at the point $\left(\alpha_{1}=\frac{3 \pi}{16}, \alpha_{2}=0\right)$ :

$$
\left.\varepsilon_{22}^{3}\left(\frac{3 \pi}{16} ; 0\right)\right|_{t=20 T}=5,53 \cdot 10^{-4} \text {. }
$$

The deformations $\varepsilon_{22}$ the time $t=13 T$ are shown in Fig. 9.

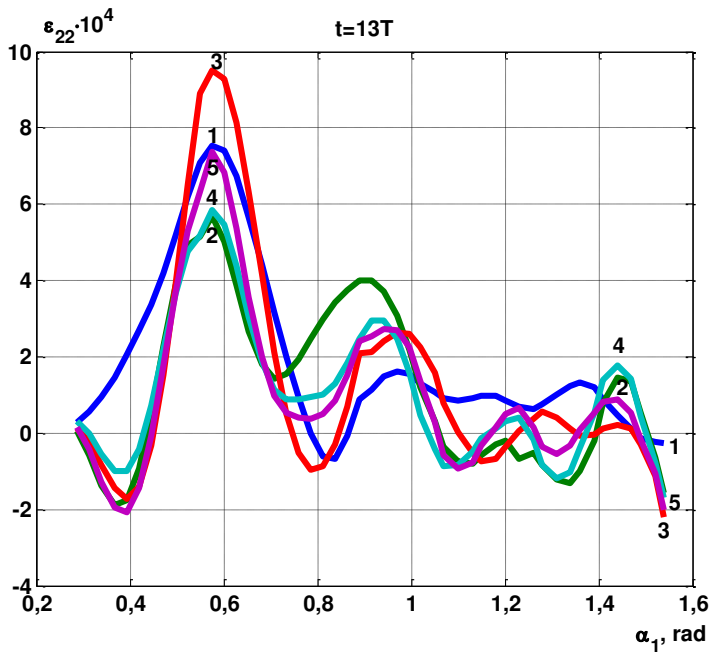

Fig. 9 Deformations $\varepsilon_{22}$ on spatial coordinate $\alpha_{1}$ in crosssection $\alpha_{2}=0$ the time $t=13 T$

The largest absolute values of the deformations $\varepsilon_{22}$ for 
given cases the time $t=13 T$ :

$$
\begin{aligned}
& \left.\varepsilon_{22}^{1}\left(\frac{3 \pi}{16} ; 0\right)\right|_{t=13 T}=7,53 \cdot 10^{-4} ;\left.\quad \varepsilon_{22}^{2}\left(\frac{3 \pi}{16} ; 0\right)\right|_{t=13 T}=5,7 \cdot 10^{-4} ; \\
& \left.\quad \varepsilon_{22}^{3}\left(\frac{3 \pi}{16} ; 0\right)\right|_{t=13 T}=9,5 \cdot 10^{-4} ; \\
& \left.\varepsilon_{22}^{4}\left(\frac{3 \pi}{8} ; 0\right)\right|_{t=13 T}=5,86 \cdot 10^{-4} ;\left.\quad \varepsilon_{22}^{5}\left(\frac{3 \pi}{16} ; 0\right)\right|_{t=13 T}=7,36 \cdot 10^{-4} .
\end{aligned}
$$

The largest value of deformation the time $t=13 T$ is obtained between the rib at the point $\left(\alpha_{1}=\frac{3 \pi}{16}, \alpha_{2}=0\right)$ :

$$
\left.\varepsilon_{22}^{3}\left(\frac{3 \pi}{16} ; 0\right)\right|_{t=13 T}=9,5 \cdot 10^{-4} .
$$

In Fig. 8-9 we can visually determine the location of the reinforcing ribs and their impact on the deformation of discretely reinforced ellipsoidal shells.

\section{Conclusions}

The problems for the deformed state of discretely reinforced ellipsoidal shells under the action of nonstationary loads are considered. The equations of oscillations for shells with corresponding boundary and initial conditions are given. The numerical algorithm based on a finite-difference approximation of the original equations in spatial and temporal coordinates is constructed to solve the problems. Numerical results for five different cases of the ribs placement on the discretely reinforced ellipsoidal shells and their comparative analysis are given.

After analyzing the calculated values for deformation $\varepsilon_{22}$, we can conclude that the largest deformation for the reinforced ellipsoidal shell is achieved at the point between the ribs $\left(\alpha_{1}=\frac{3 \pi}{16}, \alpha_{2}=0\right)$ the time $t=13 T$ for case 3 is equal to $\left.\varepsilon_{22}^{3}\left(\frac{3 \pi}{16} ; 0\right)\right|_{t=13 T}=9,5 \cdot 10^{-4}$.

Based on the obtained results, it can be concluded that of the five cases under consideration, the largest values of deformations $\varepsilon_{22}$ are achieved for case 3 . In case 3 all ribs are external placement on the ellipsoidal shell. The smallest values of deformations are observed for case 4 . In case 4 all ribs are placed internally in the shell. The largest deformation is achieved at the time $t=13 T$.

\section{Conflict of Interest}

The authors declare that they have no conflict of interest.

\section{Data Availability Statements}

The datasets generated and analyzed during the current study are available in the Science Data Bank repository, http://www.doi.org/10.11922/sciencedb.01334.(2021-11-15).

\section{References}

[1] A.E. Bogdanovich. Nonlinear problems of the dynamic buckling of reinforced laminar cylindrical shells. Soviet Applied Mechanics. 22, 745-753 (1986). DOI:https://doi.org/10.1007/BF00911327

[2] A.P. Filippov. Oscillations of deformable systems. Mashinostroyeniye, Moscow (1970).
[3] Ya. M. Grigorenko, A. Ya. Grigorenko. Static and dynamic problems for anisotropic inhomogeneous shells with variable parameters and their numerical solution (Review). International Applied Mechanics. 49, 123-193 (2013). DOI:https://doi.org/10.1007/s10778-013-0558-x.

[4] P.Z. Lugovoi, V.F. Meish, E.A. Shtantsel'. Non-stationary dynamics of inhomogeneous shell structures. Kiyevskii universitet, Kiev (2005).

[5] P. Z. Lugovoi, V. F. Meish. Dynamics of inhomogeneous shell systems under non-stationary loading (survey). International Applied Mechanics. 53, 481-537 (2017). DOI:https//doi.org/10.1007/s10778-0170833-3.

[6] N. V. Maiborodina, V. F. Meish. Forced vibrations of ellipsoidal shells reinforced with transverse ribs under a non-stationary distributed load. International Applied Mechanics 49(6), 693-701 (2013).

DOI: https://doi.org/10.1007/s10778-013-0603-9.

[7] V. F. Meish, N. V. Maiborodina. Non-axisymmetric vibrations of ellipsoidal shells under nonstationary distributed loads. International Applied Mechanics 44 (9), 1015-1024 (2008).

DOI: https://doi.org/10.1007/s10778-009-0117-7

[8] V. F. Meish, N. V. Maiborodina. Analysis of the nonaxisymmetric vibrations of flexible ellipsoidal shells discretely reinforced with transverse ribs under non-stationary loads. International Applied Mechanics 44(10), 1128-1136 (2008).

DOI:https://doi.org/10.1007/s10778-009-0125-7.

[9] V. F. Meish, N. V. Maiborodina. Stress state of discretely stiffened ellipsoidal shells under a non-stationary normal load. International Applied Mechanics 54(6), 675-686 (2018).

DOI:https://doi.org/10.1007/s10778-018-0922-y

[10] V. F. Meish, Yu. A. Meish, N. V. Arnauta. Numerical analysis of non-stationary vibrations of discretely reinforced multilayer shells of different geometry. International Applied Mechanics 55, 426-433 (2019).

DOI:https://doi.org/10.1007/s10778-019-00962-2.

[11] Xueyang Miao, Chaofeng Li, Yulin Jianga. Free vibration analysis of metal-ceramic matrix composite laminated cylindrical shell reinforced by CNTs. Composite Structures 260: 113262, 2021.

DOI:https://doi.org/10.1016/j.compstruct.2020.113262.

[12] Vuong Nguyen Van Do, Chin-Hyung Lee. Static bending and free vibration analysis of multilayered composite cylindrical and spherical panels reinforced with graphene platelets by using isogeometric analysis method. Engineering Structures 215, 110682 (2020).

DOI:https://doi.org/10.1016/j.engstruct.2020.110682

[13] V. V. Novozhilov. Fundamentals of the nonlinear theory of elasticity. Gostekhizdat, Leningrad, Moscow (1948).

[14] A.K. Pertsev, E.G. Platonov. Dynamics of shells and plates (non-stationary problems). Sudostroyeniye, Leningrad (1987) .

[15] A. A. Samarsksii. Theory of difference schemes. Nauka, Moscow (1977).

[16] N. A. Shulga, V. F. Meish. Forced vibration of three-layered spherical and ellipsoidal shells under axially symmetric loads. Mechanics of Composite Materials 39(5), 625-636 (2003).

DOI:https://doi.org/10.1023/B:MOCM.0000003294.75072.58

[17] A. V. Singh, L. Shen. Free vibration of open circular cylindrical composite shells with point supports. Journal of Aerospace Engineering 18 (2), 120-128 (2005). 